\title{
Correspondence
}

\section{Recovery and medical model - yes, science does matter}

Authors' reply We welcome Holloway's (2008) commentary on our article 'Recovery and the medical model' (Mountain \& Shah, 2008) and agree that the article raises further questions. We would, however, like to clarify that the medical model described relates to evidenced interventions practised by a range of professionals, including doctors. Doctors are certainly not the only professional group to use evidenced-based interventions. We agree that the task of answering the question 'Does recovery work?' is complex and Holloway helpfully describes some of the methodologies that may have to be deployed.

We acknowledge that by using the four elements of Resnick's work other important themes, such as meaning and self-management, were not explored. However, should Resnick's work be considered less relevant than the work of others? Of course the user perspective is central to the recovery agenda but this raises the issue of professional involvement in this agenda. In the same way that users advocate to be active participants in their care, professionals should also be actively included as equal partners to progress and mainstream recovery practice. If this is not encouraged, the view of psychiatrists as authoritarian and of professional care as 'something to get away from' could be needlessly promulgated, to the detriment of both parties and of developing recovery-oriented services. The relationship is complex, given service users' drive for selfdetermination, influenced by their experience of professional care and complicated by the potential power differential between the professionals and the often vulnerable individuals seeking their help. However, in working together to understand and develop recovery-based practice we must not let that dynamic persist. It is in developing humanistic skills while not foregoing our other professional skills that we find more in common with our patients than what separates us. This could be the common ground allowing all parties to use their unique sets of skills and capacities.

Holloway, F. (2008) Is there a science of recovery and does it matter? Invited commentary on... Recovery and the medical model. Advances in Psychiatric Treatment, 14, 245-247.

Mountain, D. \& Shah, P. (2008) Recovery and medical model. Advances in Psychiatric Treatment, 14, 241-244.

Deborah Mountain Consultant in Rehabilitation and Psychiatric Intensive Care, Royal Edinburgh Hospital, Morningside
Terrace, Edinburgh EH10 5HF, UK (email: debbie.mountain@ lpct.scot.nhs.uk); Premal J. Shah Consultant in General and Community Psychiatry and Honorary Senior Lecturer, Royal Edinburgh Hospital, Edinburgh

doi: 10.1192 /apt.14.5.398

\section{Fragile male, not fragile- $X$}

No doubt the fragile- $X$ chromosome plays a small part in male disadvantage, but the point made in my review (Kraemer, 2000), referred to by Branney \& White (2008), is that the male is biologically less resilient. Skuse et al's (1997) ingenious study of Turner syndrome shows that the $\mathrm{X}$ chromosomes inherited from mothers produce a different phenotype from paternally derived ones, but the bulk of male disadvantage probably derives from the $\mathrm{Y}$ chromosome.

Branney \& White also err in summarising my argument. It is not that 'this disadvantage is immediately mitigated once an infant's sex is known' (p. 260). On the contrary it is compounded, in ways they themselves describe in their article. Prevailing assumptions about male resilience add 'social insult to biological injury' (Kraemer, 2000: p. 1612).

Branney, P. \& White, A. (2008) Big boys don't cry: depression and men. Advances in Psychiatric Treatment, 14, 256-262.

Kraemer, S. (2000) The fragile male. BMI, 321, 1609-1612.

Skuse, D., James, R. S., Bishop, D. V. M., et al (1997) Evidence from Turner's syndrome of an imprinted X-linked locus affecting cognitive function. Nature, 387, 705-708.

Sebastian Kraemer Child and Adolescent Psychiatrist, Whittington Hospital, London N19 5NF, UK (email:@ kraemer@ doctors.org.uk kraemer@doctors.org.uk)

doi: 10.1192/apt.14.5.398a

\section{Smoking bans and clozapine levels}

The smoking ban implemented in the UK this July (Campion et al, 2008) will greatly affect psychiatric in-patients, of whom as many as $74 \%$ are smokers (Meltzer et al, 1996). Plasma concentrations of certain psychotropics are known to be affected by smoking status. Smokers are usually prescribed higher doses than non-smokers and abrupt smoking cessation will lead to high plasma concentrations and potentially more side-effects.

Clozapine plasma concentrations can rise 1.5 times in the 2-4 weeks following smoking cessation (de Leon, 2004) and in some instances by $50-70 \%$ within $2-4$ days. If baseline plasma concentrations are higher - particularly over $1 \mathrm{mg} /$ litre - the plasma concentration may rise dramatically owing to 
non-linear kinetics. If patients smoking more than 7-12 cigarettes per day while taking clozapine decide to quit, the dose may need to be reduced by $50 \%$ (Haslemo et al, 2006).

Although patients may not quit during an admission their access to cigarettes may be limited, depending on leave status or other practicalities (e.g. availability of staff to escort them off the ward). The as-required prescription of nicotine replacement therapy, although strictly speaking off-license, may help cravings but it has no effect on clozapine plasma concentration.

All patients should be forewarned that in-patient settings are now smoke-free, and clinicians need to clarify and record smoking status on admission. They will also need to monitor clozapine plasma concentrations in smokers closely during admissions and shortly after discharge. All UK assays are performed at the Toxicology Unit, Kings' College Hospital, London, and electronic access to results is possible after registration (pathologyi.t@kch.nhs. uk). Savings made by clozapine dose reductions will mitigate the additional costs incurred!

Campion, J., Checinski, K., Nurse, J., et al (2008) Smoking by people with mental illness and benefits of smoke-free mental health services. Advances in Psychiatric Treatment, 14, 217-228. de Leon, J. (2004) Atypical antipsychotic dosing: the effect of smoking and caffeine. Psychiatric Services, 55, 491-493.

Haslemo, T., Eikeseth, P. H., Tanum, L., et al (2006) The effect of variable cigarette consumption on the interaction with clozapine and olanzapine. European Journal of Clinical Pharmacology, 62 1049-1053.

Meltzer, H., Gill, B., Petticrew, M., et al (1996) Economic Activity and Social Functioning of Residents with Psychiatric Disorders (OPCS Surveys of Psychiatric Morbidity in Great Britain, Report 6). TSO (The Stationery Office)

Mohammed Ashir Specialist Registrar in Psychiatry, Tomswood Rehabilitation Unit, Whipps Cross Hospital, London E11 1NR, UK (email: mohammed.ashir@nelmht.nhs.uk); Louise Petterson Consultant in Rehabilitation Psychiatry, Goodmays Hospital, London, UK

doi: 10.1192/apt.14.5.398b

\section{Personality disorder in older adults: a pilot treatment model}

We agree with the view of Mordekar \& Spence (2008) that personality disorder does not 'burn out' with advancing age, that treatment options are limited and that more research into this area of psychiatry is needed, given that the UK has an ageing population.

The Department of Health (2001, 2003) has specifically targeted equity of access to integrated mental health services for people over 65 years of age and for people with personality disorder. In addition, NICE draft guidelines on the management of borderline personality disorder clearly support provision of integrated mental healthcare utilising a multi-model approach to psychotherapy interventions (National Institute for Health and Clinical Excellence, 2008).

We have embraced these recommendations in a pilot service for older adults diagnosable with personality disorder. The service comprises a halfday integrative group psychotherapy programme which adheres to a democratic 'mini therapeutic community' model (Pearce \& Haig, 2008).

The group is facilitated by a multidisciplinary team embedded collaboratively within the local specialist services for adults of working age with complex needs (the Oxfordshire Complex Needs Service, OCNS), local psychological services and a community mental health team for older adults. Psychodramatic techniques are used to integrate psychodynamic, cognitive and behavioural models, alongside principles of biological psychiatry, into a coherent model that is responsive to individual needs.

The experience of the facilitators who work in similar groups with both working-age and older adults suggests that the various categories of personality disorder encountered in both agegroups are similar and that both groups respond to the therapeutic model and process in a similar manner.

The OCNS treatment ethos for adults of working age is based on a recovery model, and preliminary outcome audit results of an 18-month treatment programme demonstrate psychological and socioeconomic benefits similar to the pilot for older adults (Scott \& Attwood, 2008).

There are no apparent reasons why the outcome results from the OCNS mini therapeutic community programme should not be replicated in the older adult service. Early indications from the Social Functioning Questionnaire (SFQ), the Clinical Outcomes in Routine Evaluation (CORE) system, medication audits and client satisfaction data from this pilot group are similarly optimistic. The service is committed to further evaluation and research to demonstrate effectiveness of this model over time.

This pilot programme is unique in the UK and is currently being expanded across Oxfordshire to provide a more comprehensive model of inclusive service delivery to a group of service users traditionally excluded by virtue of diagnosis and age.

Department of Health (2001) National Service Framework for Older People. Department of Health.

Department of Health (2003) Personality Disorder: No Longer a Diagnosis of Exclusion - Policy Implementation Guidance for the Development of Services for People with Personality Disorder. Department of Health.

Mordekar, A. \& Spence, S. A. (2008) Personality disorder in older people: how common is it and what can be done? Advances in Psychiatric Treatment, 14, 71-77.

National Institute for Health and Clinical Excellence (2008) Borderline Personality Disorder: Treatment and Management. NICE 